\title{
Planejamento de Recursos Humanos de Radioterapia em Hospitais do Sistema Único de Saúde (SUS).
}

\author{
Human Resource Planning Radiotherapy Hospitals in the Unified Health System \\ (SUS).
}

\section{Planificación de Recursos Humanos de Radioterapia Hospitales en el Sistema Único de Salud (SUS).}

\author{
Edward Torres MAIA ${ }^{1}$ \\ Angela Maria de Oliveira ALMEIDA ${ }^{2}$ \\ Sandro José MARTINS ${ }^{3}$ \\ Wagner de Jesus MARTINS ${ }^{4}$ \\ Gabriel Veloso MAIA \\ Moizés Ferreira Borba FILHO ${ }^{6}$
}

\begin{abstract}
RESUMO: A proposta deste estudo era averiguar se as áreas de recursos humanos de hospitais do Sistema Único de Saúde, que ofertam serviços de radioterapia à população, planejam o desenvolvimento de suas equipes de radioterapia. A metodologia aplicada neste estudo foi norteada pela seleção de cinco hospitais, considerando o critério de hospitais de médio porte que ofertam serviços de radioterapia e que serão contemplados com o recebimento de novos equipamentos por meio do Plano de Expansão de Radioterapia do SUS pelo Ministério da Saúde, além da aplicação de entrevista por meio de roteiro semiestruturado de perguntas direcionadas a 23 profissionais de radioterapia e a cinco responsáveis de recursos humanos, com posterior análise de discurso. $\mathrm{O}$ resultado obtido foi que nenhum dos hospitais visitados realizada um planejamento de desenvolvimento de seus profissionais. Há falhas no planejamento de tais profissionais aliados a desafios a serem superados. Situações que podem refletir um impacto generalizado em hospitais de radioterapia do SUS, um caminho que deve ser analisado para elevar a qualidade de serviços à população.
\end{abstract}

Palavras-chave: Recursos Humanos. Radioterapia. Saúde.

\footnotetext{
${ }^{1}$ Mestrado Profissional em Mestrado em Políticas e Gestão de CTI em Saúde pela Fundação Oswaldo Cruz, Brasil(2015) - Analista de Gestão em Saúde da Fundação Oswaldo Cruz, Brasil.E-mail:edwardmaia@ fiocruz.br

${ }^{2}$ Doutorado em Psychologie pelo Université Catholique de Louvain, Bélgica(1992) Professora Associada I aposentada da Universidade de Brasília , Brasil

${ }^{3}$ Doutorado em Pneumologia pela Universidade de São Paulo, Brasil(2005).Trabalha na McMaster University, Canadá

${ }^{4}$ Doutorado em Saúde Pública pela Escola Nacional de Saúde Pública, Brasil(2013) Coordenador de Gestão e Desenvolvimento Insti da Fundação Oswaldo Cruz -Brasília , Brasil

${ }^{5}$ Mestrado Profissional em Saúde Pública pela Fundação Oswaldo Cruz, Brasil(2015)

Analista de Gestão em Saúde da Fundação Oswaldo Cruz , Brasil

${ }^{6}$ Mestrado Profissional em Saúde Pública pela Escola Nacional de Saúde Pública, Brasil(2015)
} 
ABSTRACT: This study aimed to investigate whether the hospitals' human resources of Brazilian Health System (SUS), that offers radiotherapy services to the population, plan the development of their radiotherapy professionals. The methodology used in this study was guided by the selection of five hospitals, considering the medium-sized hospitals criteria, which offer radiotherapy services and will receive new equipment through SUS Radiotherapy Expansion Plan headed by Ministry of Health. Besides the selection, interviews through semi-structured questions were applied to 23 radiotherapy professionals and 5 responsible of human resources departments, with subsequent analysis of speech. The result was a lack of development planning for radiotherapy professionals. There are flaws regarding the planning of such professionals along with challenges to be overcome. The results may reflect a widespread impact on SUS radiotherapy hospitals, a path that must be analyzed in order to improve the quality of services to population.

Key words: Human Resources. Radiotherapy. Health.

RESUMEN: El propósito de este estudio fue investigar si las áreas de recursos humanos de hospitales del Sistema Nacional de Salud (SUS), que ofrecen a la población los servicios de radioterapia, planifican el desarrollo de sus equipos de radioterapia. La metodología utilizada en este estudio se basó en la selección de los cinco hospitales, teniendo en cuenta el criterio de los hospitales de tamaño medio que ofrecen los servicios de radioterapia y se abordará con la recepción de nuevos equipos a través del Plan de Expansión de Radioterapia del SUS por el Ministerio de Salud, así como entrevistas por medio de cuestiones con aplicación semiestructuradas dirigidas a 23 profesionales de la radiación y los cinco responsables de recursos humanos, con el posterior análisis del discurso. El resultado fue que ninguno de los hospitales visitados llevó a cabo una planificación del desarrollo de sus profesionales. Hay fallas en la planificación de este tipo de profesionales aliados a los desafíos que hay que superar. Situaciones que pueden reflejar un impacto generalizado en los hospitales de radioterapia del SUS, un camino que debe ser analizado para mejorar la calidad de los servicios a población.

Palavras clave: Recursos Humanos. Radioterapia. Salud.

\section{INTRODUÇÃO}

Um bom planejamento traz bons resultados. Esta deveria ser a máxima utilizada por empresas, organizações e administrações, em geral, quando se pretende obter desempenhos de excelência.

Quando se trata de organizações que trabalham com políticas públicas, este viés também deve ser orientado, não pela obtenção ou alcance de lucros financeiros, mas visando eficiência,

sustentabilidade econômica e lucros sociais, que contribuirão com a sociedade e sua melhoria de qualidade de vida. 
Organizações que lidam com saúde necessitam ofertar serviços ou produtos de qualidade à população, para tanto, precisam ter uma sustentabilidade em suas ações, melhorando sempre suas atividades, em uma ótica de melhoria contínua.

Paim e Teixeira (1) entendem que o planejamento em saúde se trata de ações que quando coordenadas têm como objetivo o alcance de um determinado objetivo, por meio da construção de estratégias que permitam lidar com eventuais problemas para esta política pública.

Lidar com pessoas que promovam ou conduzam as atividades, sejam elas estratégicas, táticas ou operacionais, de tais organizações em saúde é uma tarefa pertinente, pois exige um bom planejamento na área de recursos humanos para alcançar ótimos resultados institucionais, promovendo um bom ambiente e clima para se trabalhar e manter a equipe sempre motivada.

No ano de 2012, o Ministério da Saúde (MS) publicou o Plano Nacional de Saúde (2) (PNS 2012-2015) incorporando a questão do déficit da radioterapia em sua agenda, e no mesmo ano publicou o Plano de Expansão da Radioterapia no Sistema Único de Saúde (3) (PERS-SUS) para lidar com a temática e promover ações que suprissem os vazios assistenciais existentes.

Em 2013, o MS adquiriu 80 equipamentos (aceleradores lineares) para suprir o déficit existente por meio do Pregão Eletrônico de Edital número 011 (4), sendo 41 serviços de radioterapia a serem criados e outros 39 a serem expandidos até 2015. Ou seja, o cenário desenhado para o futuro de nosso país passou a ser o de entrada de diversos equipamentos sofisticados de radioterapia para suprir as necessidades detectadas anteriormente pelo TCU.

O contraponto é que cada vez mais a área de saúde necessitará de profissionais devidamente habilitados e capacitados para fazerem o devido uso dos novos equipamentos. Necessitar-se-á de profissionais competentes para assegurarem, de fato, os princípios de integralidade, universalidade e equidade sustentados pelo SUS.

A aquisição de equipamentos novos e de ponta para os hospitais requer, inquestionavelmente, profissionais preparados para exercerem suas funções, para que assim o SUS alcance seus objetivos.

Brandão e Bahry (5) afirmam que, cada vez mais, há organizações que procuram novos modelos de gestão de pessoas, "sobretudo pelo interesse por modelos de gestão que estimulem a aprendizagem, a adaptação e a inovação, capacidades consideradas essenciais numa conjuntura de aceleração do desenvolvimento tecnológico e de crescente exigência por maiores níveis de produtividade".

Desta forma, este estudo visou analisar se a área de recursos humanos de hospitais contemplados com o recebimento dos equipamentos adquiridos pelo MS possuía planos de desenvolvimento de seus profissionais da cadeia de radioterapia, com propostas de educação continuada, destacando o leque de técnicas que se fizerem necessárias em suas competências funcionais. 
Realizou-se o estudo com profissionais de radioterapia que atuavam em hospitais do SUS contemplados com o recebimento de equipamentos adquiridos pelo MS por meio do PERS.

Adotou-se como critério de seleção, hospitais considerados de médio porte (aqueles que atendem menos de 1.000 pacientes/ano) ${ }^{77}$. De um total de 146 hospitais que efetuam serviços em radioterapia e que estavam registrados no DATASUS, 14 eram considerados de grande porte e 132 situavam-se abaixo da linha de 1000 pacientes/ano.

Dentre estes 132, foram selecionados aqueles de médio porte e selecionados aqueles que fariam a expansão dos seus serviços. Em seguida, extraiu-se um hospital por região do Brasil, levando-se em consideração a facilidade de acesso (priorizou-se as capitais dos Estados).

Foram selecionados cinco hospitais, um para cada Região do Brasil. Em cada um dos hospitais foi entrevistado um representante de cada segmento da cadeia profissional da radioterapia (um médico radioterapeuta, um físico-médico, um técnico em radioterapia, um dosimetrista e um enfermeiro), além do responsável pela área de recursos humanos $(\mathrm{RH})$ de cada um deles.

Desta forma, o quantitativo de entrevistados foi de 28 profissionais: 23 profissionais de radioterapia e cinco profissionais de recursos humanos oriundos dos hospitais selecionados para a pesquisa.

No âmbito deste estudo, além da literatura técnico-científica consultada sobre a composição e competências dos profissionais que integram uma equipe ideal do serviço de radioterapia em hospitais do SUS, especialistas em radioterapia e vinculados ao Hospital Universitário de Brasília (HUB) da Universidade de Brasília (UnB), contribuíram para a definição do perfil profissional e o delineamento das respectivas competências a serem investigadas.

O instrumento utilizado para a obtenção das informações foi a entrevista, sendo que houve a aplicação de roteiro semi-estruturado de perguntas direcionadas aos profissionais de radioterapia e dos responsáveis de $\mathrm{RH}$.

1. Os profissionais de radioterapia costumam enfrentar alguma dificuldade para o efetivo exercício de suas funções?

2. Você sabe me dizer se as instituições hospitalares asseguram cursos de capacitação para os profissionais da radioterapia?

Sim - Não: justifique, por favor.

3. E no seu hospital, como são ofertados os cursos e em qual periodicidade?

\footnotetext{
${ }^{7}$ Definição em consonância com o Banco de dados do Sistema Único de Saúde (DATASUS) do ano de 2012 (compilados em junho/2013).
} 
4. Você considera que é difícil para os profissionais da radioterapia obter a capacitação necessária para o exercício de suas funções?

Aos responsáveis pela área de RH dos hospitais visitados foram realizadas as seguintes perguntas:

1. Quanto aos profissionais de radioterapia que compõem a equipe deste hospital, eles já chegam devidamente capacitados ou há um plano de desenvolvimento para eles?

2. O hospital oferece capacitações periódicas aos profissionais das equipes de radioterapia deste hospital?

3. A área de recursos humanos deste hospital encontra dificuldades para promover a capacitação dos profissionais de radioterapia? Quais seriam elas?

4. Vocês contam com instituições parceiras que promovam a capacitação dos profissionais de radioterapia neste hospital? Se sim, quais são estas instituições?

Após as respostas terem sido gravadas, elas foram degravadas e passaram por uma análise de discurso das respostas elencadas.

Todas as entrevistas foram consentidas por meio da assinatura do Termo de Consentimento Livre e Esclarecido (TCLE) aprovados pelo Comitê de Ética da Escola Nacional de Saúde Pública (ENSP) da Fundação Oswaldo Cruz.

Para efeito das investigações desenvolvidas neste estudo, os profissionais de radioterapia no Brasil foram elencados como sendo a equipe de radioterapia considerada ideal que houvesse nos hospitais, em termos de categorias profissionais: Médico Radioterapeuta (MRD); Físico Médico (FMR); Dosimetrista Médico (DMR); Técnico ou Tecnólogo em Radioterapia (TRD); e Enfermeiro em Radioterapia (ERD).

Um adendo quanto ao profissional dosimetrista médico, pois a formação deste profissional no Brasil ainda não se encontra oficialmente instituída para efeitos de reconhecimento e validação do diploma, conforme verificado no Sistema e-MEC do Ministério da Educação, e há uma tendência de hospitais adequarem profissionais, por meio de uma capacitação em serviço (supervisionadas geralmente pelo FRD), a exercerem a função deste profissional, conforme expresso por Maia (6). 
Para poder verificar a qualificação desejada do profissional para exercer a profissão no âmbito da radioterapia, o quadro 1 exemplifica a necessidade por categoria.

Quadro 1: Relação de profissionais da cadeia de radioterapia e a suas qualificações desejadas.

\begin{tabular}{|c|c|}
\hline PROFISSIONAL & QUALIFICAÇÃO DESEJADA \\
\hline \multirow{3}{*}{ MRD } & Graduação em medicina \\
\hline & + \\
\hline & Pós-graduação em oncologia ou em radioterapia \\
\hline \multirow{3}{*}{ FMR } & Graduação em física ou física médica \\
\hline & + \\
\hline & Pós-graduação em radioterapia \\
\hline \multirow{3}{*}{ DMR } & Graduação com Bacharelado \\
\hline & + \\
\hline & $\begin{array}{l}\text { Certificado Internacional ou Pós-graduação em oncologia ou } \\
\text { radioterapia }\end{array}$ \\
\hline TRD & $\begin{array}{l}\text { Formação técnica (especialização em radioterapia, em qualificação, } \\
\text { do Conselho Nacional de Técnicos em Radiologia de } 1200 \text { horas para } \\
\text { técnico em radiologia e } 2400 \text { horas para tecnólogo em radiologia }\end{array}$ \\
\hline \multirow{3}{*}{ ERD } & Graduação em enfermagem \\
\hline & + \\
\hline & Pós-graduação em oncologia ou em radioterapia \\
\hline
\end{tabular}

\section{RESULTADOS}

Os resultados apontam para falhas encontradas no planejamento de capacitação dos profissionais de radioterapia e dificuldades de gestão a serem superados nos hospitais visitados.

\section{Falhas no planejamento de capacitação dos profissionais de radioterapia.}

Neste estudo, detectou-se que todos os responsáveis pela área de recursos humanos dos hospitais entrevistados afirmaram que não havia plano de desenvolvimento para os profissionais de radioterapia de seus hospitais.

Os responsáveis pelos recursos humanos dos hospitais das Regiões S e SE (hospitais privados) mencionaram que há capacitações esporádicas ofertadas pelos hospitais aos profissionais de radioterapia, entretanto, não há um plano de desenvolvimento a curto, médio ou longo prazo.

Os profissionais de radioterapia de hospitais do SUS que mais se capacitam eram os MRDs e os FMRs. Tais profissionais já chegam bem melhores formados em função da residência do que os outros profissionais da cadeia. 
As categorias profissionais de MRDs e de FRDs possuem mais eventos científicos vinculados à área e desta forma, são os profissionais que mais se capacitam na cadeia de radioterapia.

Constatou-se a prática de treinamentos que são obrigados/regulados por lei/norma da CNEN (Resolução N130/2012) (7) a serem realizados para a categoria profissional de técnicos/tecnólogos, cuja periodicidade é anual. Este treinamento é realizado pelo Supervisor de Proteção Radiológica do Serviço de Radioterapia, geralmente é o profissional físico-médico que são certificados.

Além deste treinamento de uma categoria profissional, há outros dois treinamentos geralmente: o inicial ofertado pelos próprios colegas para conhecer o funcionamento dos hospitais e padronizar o atendimento ao público, além da capacitação provida pelos próprios fornecedores das empresas dos equipamentos de radioterapia que são adquiridos pelos hospitais.

Desta forma, a percepção geral deste estudo é que os hospitais não apresentam propostas proativas de treinamentos aos seus profissionais da cadeia de radioterapia, de forma sustentável, sendo que selecionam ou recrutam profissionais de radioterapia já devidamente capacitados para compor as equipes.

\section{Dificuldades de Gestão}

Para efeitos de entendimento, algumas características dos profissionais deste estudo foram sintetizadas no quadro abaixo, representando, assim, a relação entre os profissionais da cadeia de radioterapia e as suas qualificações que seriam consideradas desejadas para o exercício de suas funções.

A partir das declarações de profissionais das áreas de recursos humanos e de radioterapia, foram reveladas diversas dificuldades referentes à promoção da capacitação dos profissionais de radioterapia. O quadro 2 sintetiza as dificuldades mencionadas durante as entrevistas.

Quadro 2: Dificuldades percebidas pelos entrevistados relativas à capacitação dos profissionais de radioterapia

\begin{tabular}{|l|l|}
\hline \multicolumn{1}{|c|}{ Dificuldades } & \multicolumn{1}{c|}{ Âmbito ou natureza } \\
\hline Alinhamento entre os setores & Falta de comunicação entre a área de RH e os profissionais de radioterapia \\
\hline Inexistência de incentivos & Utilização de recursos próprios para a sua capacitação \\
\hline Barreiras legais & Dificuldades na aplicação de recursos públicos \\
\hline Organização do trabalho & Falta de tempo/horário dos profissionais de radioterapia \\
\hline
\end{tabular}




\begin{tabular}{|l|l|}
\hline Investimento em pessoal & Escassez de profissionais \\
\hline Cooperação/Parceria externa & Falta de instituições parceiras \\
\hline
\end{tabular}

Dentre os cinco profissionais entrevistados responsáveis pela área de RH dos hospitais de radioterapia, três apresentaram, nos discursos, informações de que não havia comunicação direta entre eles e os profissionais de radioterapia. Dos hospitais em que havia comunicação, considerase como intermediador o profissional físico-médico, ponte entre os profissionais e a área de recursos humanos.

Nos discursos de todos os responsáveis por recursos humanos, verificaram-se percepções ou expectativas distintas. De um lado, os profissionais de radioterapia que gostariam de ser mais valorizados e ter capacitações periódicas; e de outro, os responsáveis pelos departamentos/setores de recursos humanos que não apresentavam planos de desenvolvimento para seus profissionais de radioterapia e alegavam a inércia dos profissionais na busca por capacitação junto aos setores pertinentes.

Tais fatos demonstram falhas no alinhamento entre as áreas, mais evidenciadas como problemas de comunicação entre os setores de recursos humanos e os profissionais de radioterapia na maioria dos hospitais.do SUS visitados nesta pesquisa.

O discurso da maioria dos profissionais de radioterapia entrevistados apresentou problema relacionado à capacitação por conta própria, em função de utilização/aplicação de seus recursos financeiros, apesar do aval dos hospitais. E isso era comum a todos os profissionais, visto que não havia incentivos ofertados pelos hospitais à força de trabalho.

Um dos itens que apareceu como dificuldade para o hospital da região Centro-Oeste (hospital público) foi referente às barreiras legais existentes quanto à aplicação de recursos nas capacitações em profissionais de radioterapia que não eram servidores públicos.

Detectou-se também uma dificuldade sobre tempo/horário, que muito decorre em função de eventos de capacitação serem realizados em outras cidades ou então por estarem em outros empregos além dos que já possuem no hospital, inviabilizando a participação.

Capacitar a equipe sem haver um bom quadro de funcionários é considerado um problema também em função da necessidade de se manter a oferta de serviços nos hospitais. A cadeia ideal de profissionais de radioterapia envolve cinco especialidades necessárias em países de primeiro mundo: MRDs, FRDs, DMRs, TRDs e ERDs. Esta pesquisa detectou a insuficiência de profissionais nos hospitais visitados, em particular do profissional DMR em 2 hospitais (Regiões SE e N).

Em função da escassez ou limitação no quantitativo de profissionais de cada um dos setores, o profissional que seria coberto por um colega da mesma categoria não pode se ausentar para se 
capacitar porque impactaria o serviço ofertado à população em função de não haver algum outro colega para suprir a sua ausência. A situação é pior ainda quando fosse para que o profissional se capacitasse fora de sua cidade.

Constatou-se também, nesta pesquisa, que nenhum dos hospitais visitados (públicos e privados) contavam com instituições parceiras que promovessem a capacitação de seus profissionais de radioterapia. Tal fato demonstra a fraca articulação entre centros formadores e a busca contínua por melhorias profissionais da força de trabalho da radioterapia em hospitais do SUS.

\section{DISCUSSÃO}

Os resultados apontaram para constatações e desafios na área de gestão de pessoas. Fernandes et al (8) defende que os trabalhadores são administrados por pessoas que ocupam cargos gerenciais, embasados por diretrizes e práticas oriundas de órgãos competentes, e que é responsável diretamente pela liderança, motivação, avaliação e recompensação. O propósito dado pelo autor é de elevar a eficiência no desenvolvimento de suas atividades, proporcionando, assim, maior efetividade nos resultados.

Tal posicionamento do autor deveria ser uma regra a ser seguida dentro da área de recursos humanos dos hospitais de radioterapia. $\mathrm{O}$ alinhamento gerencial entre o responsável pelo $\mathrm{RH}$ e o responsável pela equipe de radioterapia deve ser uma constante que visa a elevação nos resultados, por meio da capacitação de seus funcionários e alta motivação.

Uma vez que houver tal alinhamento, os resultados começam a melhorar, elevando a eficiência, a eficácia e a efetividade das ações dos profissionais de radioterapia. $\mathrm{O}$ fato de não haver um planejamento de desenvolvimento da força de trabalho, como é o resultado encontrado nesta pesquisa, vai de encontro às melhores práticas que existem.

Pierantoni et al (9) afirmam que no setor de saúde, há uma histórica negligência e ausência de políticas para RH devido a fatores como não valorização do papel dos profissionais. Os autores ressaltam a importância do planejamento e o gerenciamento de recursos humanos da saúde como pontos essenciais para o eventual equacionamento de problemas vinculados à baixa capacidade de implementação das políticas públicas.

A proposta de fortalecimento da capacidade de planejamento e gerenciamento de RH no setor de saúde deve ser um fator essencial a ser tratado nos hospitais de radioterapia do SUS. A falta de planejamento constatada impacta diretamente nos resultados dos hospitais e na qualidade dos serviços ofertadas à população do país.

\section{CONCLUSÃO}

Dado o cenário de entrada de novos equipamentos no país, e considerando que há hospitais em que a radioterapia é um novo campo de atuação, há desafios a serem superados. 
Desafios como manter o corpo de profissionais dos hospitais de radioterapia do SUS devidamente capacitados (periodicamente e de forma sustentável) e promover uma cultura de planejamento de desenvolvimento das equipes de radioterapia nos hospitais foram objetos desta pesquisa realizada.

Contudo, o presente estudo demonstrou, por meio da percepção dos participantes, que há falhas no planejamento de desenvolvimento dos profissionais de radioterapia pelo $\mathrm{RH}$ dos hospitais da amostra.

Com o propósito de ofertar serviços de qualidade à população, os profissionais de radioterapia necessitarão ter uma educação continuada e de qualidade para garantir sempre o melhor nível de serviço. Para isso, o planejamento de RH será essencial.

Assim, seria oportuno também que os setores de RH dos hospitais aplicassem metodologias contemporâneas de gestão de pessoas, objetivando motivar suas forças de trabalho, conjugando todas as competências técnicas necessárias para as realizações dos serviços de radioterapia, de forma a alcançar melhores resultados na ótica dos hospitais e na ótica dos pacientes.

\section{REFERÊNCIAS}

1. Paim JS, Teixeira CF. Política, planejamento e gestão em saúde: balanço do estado da arte. Rev. Saúde Pública [online]. 2006, vol.40, n.spe, pp. 73-78. Acesso em 12 out. 2015. Disponível em: 〈http://dx.doi.org/10.1590/S0034-89102006000400011〉.

2. Ministério da Saúde (BR). Secretaria Executiva. Subsecretaria de Planejamento e Orçamento. Plano Nacional de Saúde: PNS: 2012-2015. Brasília, DF: Ministério da Saúde, 2011b. (Série B. Textos Básicos de Saúde). Disponível em: 〈http://conselho.saude.gov.br/ biblioteca/Relatorios/plano_nacional_saude_2012_2015.pdf >. Acesso em 05 jan. 2014.

3. Ministério da Saúde (BR). Gabinete do Ministro. Portaria $n^{\circ}$ 931, de 10 de maio de 2012. Institui o Plano de Expansão da Radioterapia no Sistema Único de Saúde (SUS). Brasília, DF: Ministério da Saúde, 2012. Disponível em: <http://bvsms.saude.gov.br/bvs/saudelegis/gm/2012/ prt0931_10_05_2012.html >. Acesso em 05 de jan. 2014.

4. Ministério da Saúde (BR). Secretaria Executiva. Departamento de Logística em Saúde. Edital do pregão presencial número 11/2013: processo n 25000.096286/2012-93. Este Pregão tem por objeto a implementação de 80 soluções de radioterapia, compreendendo equipamentos, projetos básico e executivo, apoio técnico ao acompanhamento e fiscalização de obras, conforme especificações técnicas básicas e técnicas obrigatórias consignadas nos Anexos deste Edital, para consolidar o Plano de Expansão da Radioterapia no Sistema Único de Saúde - SUS previsto na Portaria MS nº 931 de 10 de maio de 2012 Brasília, DF: Ministério da Saúde, 2013. Disponível 
em: 〈http://www.sbradioterapia.com.br/pdfs/pregao-presencial.pdf $>$. Acesso em 05 dez. 2013.

5. Brandão H, Bahry CP. Gestão por competências: métodos e técnicas para mapeamento de competências. Brasília, DF: Revista do Serviço Público, 2005. Disponível em: <www.spell.org. br/documentos/download/35846 $>$. Acesso em 01 mar. 2014.

6. Maia ET. Mapeamento de competências de profissionais de radioterapia em hospitais do SUS. 2015, 130 f: il. Dissertação (Mestrado em Política e Gestão de Ciência, Tecnologia e Inovação em Saúde) - Escola Nacional de Saúde Pública - Fundação Oswaldo Cruz. Rio de Janeiro, 2015. Disponível em: 〈bvssp.icict.fiocruz.br/lildbi/docsonline/get.php?id=4283>. Acesso em 01 nov. 2014.

7. Ministério da Ciência, Tecnologia e Inovação (BR). Comissão Nacional de Energia Nuclear. Resolução CNEN número 130, de 31 de maio de 2012. Dispõe sobre os requisitos necessários para a segurança e a proteção radiológica em Serviços de Radioterapia. Brasília, DF: Comissão Nacional de Energia Nuclear, 2012. Disponível em: <http://memoria.cnen.gov.br/Doc/ pdf/Legislacao/RS CNENCD 130 2012.pdf>. Acesso em 05 mar. 2014.

8. Fernandes LCL, Machado RZ, Anschau GO. Gerência de serviços de saúde: competências desenvolvidas e dificuldades encontradas na atenção básica. Ciênc. saúde coletiva [online]. 2009, vol.14, suppl.1, pp. 1541-1552. ISSN 1678-4561. Disponível em: <http://www.scielo.br/pdf/csc/ v14s1/a28v14s1.pdf>. Acesso em 01 out. 2015.

9. Pierantoni C, França T, Varella TC. Recursos humanos e gestão do trabalho em saúde: da teoria para a prática. In: FALCÃO, A. et al. (Org.). Observatório de recursos humanos em saúde no Brasil: estudos e análises. v. 2. Rio de Janeiro: Fiocruz, 2004, p. 51-70. Disponível em: <http://www.obsnetims.org.br/uploaded/16_5_2013_0_Recursos_Humanos_e_gestao.pdf $>$. Acesso em 01 out. 2015.

Artigo apresentado em 01-11-14 Artigo aprovado em 07-05-15 Artigo publicado no sistema em 22-12-15 